Presented at the 31st IEEE Conf. on Decision and Control (CDC)

Tucson, Arizona, December 16-18, 199:

\title{
MONOCULAR 3-D VISUAL TRACKING OF A MOVING TARGET BY AN EYE-IN-HAND ROBOTIC SYSTEM
}

\author{
N.P.Papanikolopoulos* , B. Nelson**, and P.K. Khosla** \\ "Department of Computer Science \\ University of Minnesota \\ 200 Union St. SE \\ Minneapolis, MN 55455 \\ ** Department of Electrical and Computer Engineering \\ The Robotics Institute \\ Carnegie Mellon University \\ Pittsburgh, Pennsylvania 15213
}

\begin{abstract}
This paper epplies the fremowat of controlled ective vision to the problem of monoculer full

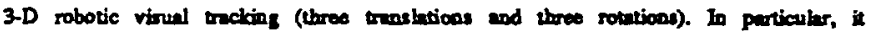
deconotrate full 3-D tracking of a moving teget by a monocular hed-oye system. A single

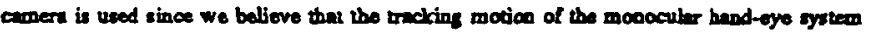
cas be ued to cresce virtul steroo langes. A simple deptive scheme is proposed and the

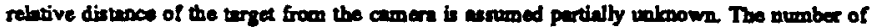

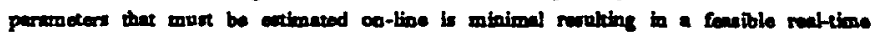
implementution of the scberne. Moreover, the atroas coupling of the rotationel and

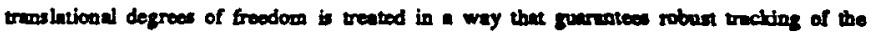
object The limitnions of our approweh are diecused and the results trom the applicapion of our scheme to the TRONKABOT system (a set of throe PUMAS60's menipulemers) are preanted.
\end{abstract}

\section{Introduction}

This paper deals with the problem of 3-D robotic visual tracting of targets whose motion consists of 3-D transiational and rotational components. The visual tracking is accomplished through a camera mounted on the robot that computer the displacements of several features that belong to the target. These visual measurements are fed to an adaptive control algorithm that provides the input to 2 cartesian robot contsol scheme after each measurement Numerical issues related to the strong coupling of the rotational and translational degrees of freedom are treated in a way that guarantees trebing of the. object. A single camerais used instead of a binocular system because one of our main objectives is to demonstrate that relatively unsophisticated offthe-self hardware can be used to solve the 3-D trating problem if the proper modeling and control issues are addressed.

The major differences of our algorithms from sirilar research efforts $[1,2,3,4,5]$ are the use of a single moving cameres the ability to compensate for inaccurale camera parameters and unknown depth (distance of the target with respect to the camera frame), the full 3-D tracking ability, the small number of parameters that are estimated on-line, and the insegration of the characteristes of the motion detection algorithm into the mathematical model for tracking. These differences allow the use of the proposed algorithms in poody cali arated spaces or

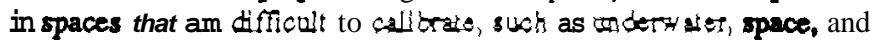
nuclear sites. This paper extends our previous work $[6,7,8]$ in controlled active vision by allowing tracking of full 3-D motion (translations and rotations) and by d u cing the number of parameters that should be estimated on-line. Experimental resules arc presented to show the strengths and the weaknesses of the proposed approach The experiments am performed on the TROIKABOT multi-robotic system which operales under the CHIMERA11 real-time operating system. The TROIKABOT system consists of three PUMA560's. One PUMA carries the camera while another holds the target.
The organization of this paper is as follows: Section 2 describes the mathematical framework under which our problem is solved. The control, filtering, and extimation strategies are discussed in Section 3. The experimental results are presented in Section 4. Finally, in Section 5, the papa is summarized.

\section{Modeling of the 3-D Robotic Visual Tracking Problem}

This section describes the mathematical modeling of our problem. W e assume a pinhole camera model with a frame $\boldsymbol{R}_{s}$ placed at the focal point of the lens. Consider a target with a feature located \pm a point $\mathbf{P}$ with coordinates $\left(X, Y_{2}, Z\right)$ in $\mathrm{R}$, Moreover, the camera moves with a translational velocity $\mathbf{T}=\left(T_{x}, T_{y}, T_{z}\right)^{r}$ and with an angular velocity $R=\left(R_{z}, R_{y}, R_{z}\right)^{r}$ with resper to the camera frame $R$, Since the camera and the target are moving simultaneausly. we can write (using the approach described in (91) the following equations for one feature point ( $d$ is a delay factor $(d \mathrm{E}(1,2, \ldots)$ ), $f$ is the focal length of the camera, $s_{x}, s_{y}$ am the dimensions (mra/pixel) of the camera's pixels, $(x, y)$ are the inage coordinates of the projection of the feartre point $\mathbf{P}$ on the image plane, Tis the sampling period, $q^{-1}$ is the backwand shift qperator, and $\mu_{0}(k)$ and $\psi_{0}(k)$ are the components of the optical flow induced $a$ the time instan $k$ by the motion of the object):

$$
\begin{aligned}
x_{F}(k+1)=A_{F} & (k) x_{F}(k)+B_{F}(k-d+1) u(k-d+1)+E_{F}(k) d,(k) \\
& +H_{F}(k) v_{F}(k)
\end{aligned}
$$

where" $\quad A_{F}(k)=H_{F}(k)=I_{2}, \quad E_{F}(k)=T I_{2}, \quad x_{F}(k) \in R^{2}, \quad d_{F}(k) \in R^{2}$, $u(k) \in R^{6}$, and $v_{F}(k) \in R^{2}$. The marrix $B_{F}(k) \in R^{2 \times 6}$ is:

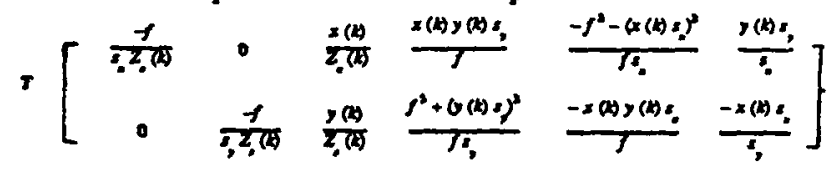

The vector $x_{F}(k)=(x(k), y(k))^{T}$ is the state vector, $u(k)=\left(T_{x}(k), T_{y}(k), T_{z}(k), R_{x}(k), R_{y}(k), R_{z}(k)\right)^{T}$ is the control input vector, $d_{F}(k)=\left(u_{e}(k), v_{0}(k)\right)^{T^{T}}$ is the exogenous deterministic disturbances vector, and $v_{Y}(k)=\left(v_{x}(k), v_{y}(k)\right)^{r}$ is the white noise vector. The measurement vector $\left.y_{F}(k)=\sigma_{x}(k), y_{y}(k)\right)^{T}$ for this feature is given by:

$$
y_{F}(k)=C_{F} x_{F}(k)+w_{F}(k)
$$

where $w_{y}(k)=\left(w_{x}(k), w_{y}(k)\right)^{T}$ is a white noise vector $\left(w_{F}(k)-N(0, W)\right.$ ) and $C_{F}=I_{2}$. The measurement vector is computed using the SSD algorithm which is described in [9].

\footnotetext{
"The symbol I denotes the ideatity marix of arder $n$.
} 
One fearure point is not enough to determine the control input yector $\checkmark(k)$. The reason is that the number of system outputs is less than the number of control inputs. Thus, we are obliged to consider more points in our model. In order to solve for the control input that will be sent to the manipulator, it can be shown that a least three non-collinear fearure points arc neaded. The reason for the non-collinearity requirement is investigatedin [IO]

The state-space model for $\boldsymbol{M}(\boldsymbol{M} \geq 3)$ fearure points can be written as: $x(k+1)=A(k) x(k)+B(k-d+1) u(k-d+1)+E(k) d(k)+\mathrm{H}(k) \vee(k) \quad(3)$ where $A(k)=H(k)=L_{2 H}, E(k)=T I_{2 \text { sr }} \geq(k) E R^{2 M}, d(k) E R^{2 M}$, and $\nabla(k) E R^{2 M}$. The matrix $\mathrm{B}(k)$ E $R^{2 M \times 6}$ is:

$$
\mathbf{B}(k)=\left[\begin{array}{c}
\mathrm{B}_{\mathrm{F}}^{(1)}(k) \\
\cdots \\
\mathbf{B}_{\mathrm{F}}^{(M)}(k)
\end{array}\right] .
$$

The superscript $(j)$ denotes each one of the feartre points $((j)$ E $\{(1), \ldots,(M)\})$. The vector $x(k)=\left(x^{(1)}(k), y^{(1)}(k), \ldots\right.$, $\left.x^{(M)}(k), y{ }^{(M)}(k)\right)^{T}$ is the new state vector. and $\mathbf{v}(k)=\left(v_{x}^{(1)}(k)\right.$, $\left.v_{Y}^{(1)}(k), \ldots, v_{x}^{(M)}(k), v_{y}^{(M)}(k)\right)^{T}$ is the new white noise vector. The new measurement vector $y(k)=\hat{y}_{x}^{(1)}(k), y_{y}^{(1)}(k), \ldots, y_{x}^{(m)}(k)$, $\left.y_{y}{ }^{(H)}(k)\right)^{T}$ for $\boldsymbol{M}(M \geq 3)$ feaflues is given by:

$$
y(k)=\mathbf{C X}(k)+w(k)
$$

Where $w(k)=\left(w_{x}^{(1)}(k), w_{y}^{(1)}(k), \ldots, w_{z}^{(M)}(k), w_{y}{ }^{(k)}(k)\right){ }^{T}$ is the new white noise vector $\left(\boldsymbol{F}(k) \underline{y}_{N}(0, W)\right)$ and $\mathbf{C}=\mathbf{I}_{2 M}$

We can combine quations (3)-(4) into a MMO (Multi-Input MultiOutput) model:

$$
\left(1-2 q^{-1}+q^{-2}\right) \text { y }(k)=\mathrm{B}(k-d) \mathrm{u}(\mathrm{C}-d)-\mathrm{B}(k-d-1) \mathrm{u}(k-d-1)+\mathrm{n}(k)
$$

Where $\square(k)$ is the white noise vector. The new white noise vector $n(k)$ corresponds to the measurement noise, to the modeling errors, and to the noise introduced by inaccurate robot control. If we sssume $B(k-d)=B(k-d-1)$, then (5) can be repriten as a MMO ARX (AwoRegressive with auxiliary input) model. This model consists of 2M MISO (Multi-Input Single-Outpur) ARX models. In addition, the new model's equation is:

$$
\left(1-2 q^{-1}+q^{-2}\right) \text { y }(k)=\mathbf{B}(k-d) \Delta \mathbf{u}(k-d)+\mathbf{n}(k)
$$

where $\Delta u(k-d)$ is defined as:

$$
\Delta u(k-\theta)=\mathbf{u}(k-\phi)-\mathbf{u}(k-d-1) \text {. }
$$

In the next section, we present the control and estimation techniques for the 3-D visual tracking problem.

\section{Control and Estimation}

The control objective is to move the manipulator in a such a way that the projections of the selected features on the image plane move to some desired positions $a$ stay at their desired positions while the larget is moving. This section examines the control strategies that realize this motion and the estimation scheme used to estimate the unknown paraneters of the model. Some implementation issues are also dis. cussed.

Adaptive control techniques can be used for visual servoing around a moving object when the depth of the object is not precisely known. Adaptive control techniques are used for the recovery of the components of the transiational and rotational velocity vectors, $T(k)$ and $R(k)$, respectively, and ar based on the estimated and not the actual values of the system's parameters. This approach is called certainty equivalence adaptive control [11]. A large number of algorithms can te generated depending on which parameter estimation scheme is used and which control law is chosen. The rest of this section is devoted to a detailed description of the control and estimation schemes.

\subsection{Selection of an Efficient Control Law}

The control objective is to track the motion of certain features of the target and place their projections on the image plane at some desired positions. The tracking of the features' projections is realized by an appropriate motion of the robot-camera system. A simple control law can be derived by the minimization of a cost function that includes the fearre positional tror, the control signal. and the change in the control signal:

$$
\begin{aligned}
J(k+d)= & {\left[y(k+d)-y^{*}(k+d)\right]^{T} Q\left[y(k+d)-y^{*}(k+d)\right] } \\
& +\mathbf{u}^{T}(k) \mathrm{Lu}(k)+\Delta \mathbf{u}^{T}(k) \mathbf{L}_{\mathbf{d}} \mathrm{AU}(k) .
\end{aligned}
$$

The vector $y^{\prime}(k)$ represents the desired positions of the projections of the $H(M \geq 3)$ fearures on the image plane. In our experiments, the vector $\mathbf{y}^{*}(k)$ is known a priori and is constant over time. By placing waghts on the control signal. the change in the control signal, and the error, we can choose how much emphasis the controller is to place on minimizing each of the thre quantities. Including the control signal and the change in the control signal in the cost function described by (8) causes the control input signal to be bounded and feasible. This is in agrement with the structural and operational characteristics of the robotic system and the vision algorithm. A robotic system cannot track signals that command large changes in the fearures' image projections during the sampling interval T. In addition. our optical flow algorithm cannot detect displacements larger than 28 pixels per sampling interval T. The term $\Delta u^{T}(k) L_{d} \Delta u(k)$ of the cost function (8) introduces an integral term in the control law. This term is desirable since our mathematical model (3) has a deterministo disturbances component. one prodern of the introduction of an integral term in the control law is the possible saturation of the control inputs. In order to compensate for this problem, one should turn off the integrator whenever s saturation of the control inputs oceurs.

The control law is derived from the minimization of the cost function (8) by taking the derivarve of $J(k+\phi)$ with respect to the vector $u(k)$ and combining the resulting prosion with the system model equation (5). The resulang control law is:

$$
\begin{gathered}
\mathbf{u}(k)=-\left[\mathrm{B}^{T}(k) \mathrm{QB}(k)+\mathrm{L}+\mathrm{L}_{\mathbf{d}} \boldsymbol{T}^{-1}\left[\mathrm{~B}^{T}(k) \mathrm{Q}(\{(d+1) \mathbf{y}(k)\right.\right. \\
\left.-\mathbf{y}^{*}(k+\delta)-\mathrm{d} \mathbf{y}(k-1)\right)-d \mathrm{~B}(k-d) \mathbf{u}(k-d) \\
\left.\left.+\sum_{m=d-1} \mathrm{~B}(k-m) \mathbf{u}(k-m)\right)-\mathbf{L}_{\mathbf{d}} \mathbf{u}(k-1)\right] .
\end{gathered}
$$

Feddems and Lee [3] proposed a similar control law for the robotic visual tracking problem. The main difference of our control law is that, instead of imposing constraints on the optical flow induced by the camera motion (image plane space). we impose constraints on the components of $\mathbf{u}(k)$ of the required camera trabing motion (camera frame space). In this way, we directly control the magnitudes of the control signal and the control signal change. This fact results in a control law that is more robust and feasible than the one proposed in [3]. The desigr pamerts in our control law the elements of the matrices $Q, L$, and $L_{d}$ Often, we set $L_{\text {or }} L_{d}$ to zero. Inmostofthe experiments, we set $\mathrm{L}=0$ and $\mathrm{L}, \neq 0$ in order to achieve $a$ fast and bounded response. If the matrix $\mathrm{B}(k)$ is full rank then the matrix $\left.\mathbf{B}^{\mathbf{T}}(k) \mathrm{QB}(k)+\mathrm{L}+\mathrm{L}_{\mathbf{d}}\right]$ is invertible. The marrix $\mathrm{B}(k)$ is singular when the $M$ feature points are collinear $[3,10]$. An extensive study of other conditions which make $B(k)$ singular can be found in [9].

By selecting $\mathrm{L}, \mathrm{L}_{\boldsymbol{d}}$ and $\boldsymbol{Q}$, om con place more or less emphasis on the control input, the control input change, and the servoing error. There is no standard procedire for the selection of the elements of these matrices. One technique is the optimization approsch [12]. 
If we want to include the noise of our model and the inaccuracy of the B (k)matrix in out control law. the control objective (8) becomes:

$$
\begin{aligned}
J(k+d)= & E\left\{\left[y(k+d)-y^{*}(k+d)\right]^{T} Q\left[y(k+d)-y^{*}(k+d)\right]\right. \\
& \left.+\mathbf{u}^{T}(k) L u(k)+\Delta u^{T}(k) \mathbf{L}_{d} \Delta u(k) \mid F_{k}\right\}
\end{aligned}
$$

where the symbol $E(X)$ denotes the expected value of the randam variable $\mathbf{X}$ and $\boldsymbol{F}_{\boldsymbol{k}}$ is the sigma algebra generated by the past measurements and the past control inputsup to time \& The new control law is:

$$
\begin{aligned}
\mathbf{u}(k)=- & {\left[\hat{\mathbf{B}}^{T}(k) \mathrm{Q} \hat{\mathbf{B}}(k)+\mathrm{L}+\mathbf{L}_{\mathbf{d}} \mathbf{J}^{-1} \hat{\mathrm{B}}^{T}(k) \mathrm{Q}(\{(d+1) \mathbf{y}(\mathrm{k})\right.} \\
& \left.-\mathbf{y}^{*}(k+d)-d y(k-1)\right\}-d \hat{\mathbf{B}}(k-d) \mathbf{u}(k-a) \\
& \left.\left.+\sum_{m=d-1} \hat{\mathrm{B}}(k-m) \mathrm{u}(k-m)\right\}-\mathbf{L}_{\mathbf{d}} \mathbf{u}(k-1)\right]
\end{aligned}
$$

where $\hat{\mathbf{B}}(k)$ is the estimated value of the matrix B $(k)$. The matrix $\hat{\mathbf{B}}(k)$ is dependent on the estimated values of the fearures' depth $\hat{Z}_{j}^{(I)}(k)$ $((j)$ E $((1), \ldots,(M)\})$ and the coordinates of the fearres' image projections. In particular, the marrix $\hat{B}(k)$ is defined as follows:

$$
\hat{\mathbf{B}}(k)=\left[\begin{array}{c}
\hat{\mathbf{B}}_{\mathbf{F}}^{(1)}(k) \\
\cdots \\
\hat{\mathbf{B}}_{\mathbf{F}}^{(m)}(k)
\end{array}\right]
$$

where $\hat{\mathbf{B}}_{\mathbf{F}}^{(j)}(\mathbf{k})$ is:

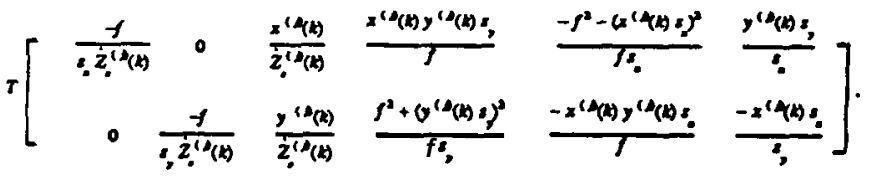

In the experiments. the delay factor $\mathrm{d}$ is $\mathbf{2}$, so the control law (11) becomes:

$$
u(k)=-\left[\hat{B}^{T}(k) Q B(k)+L+L J^{-1}\left[\hat { B } ^ { T } ( k ) Q \left\{\left(3 y(k)-y^{*}(k+2)\right.\right.\right.\right.
$$

$$
\left.-2 y(k-1)\}-2 \dot{\mathbf{I}}(k-2) \mathbf{u}(k-2)+\dot{B}(k-1) \mathbf{u}(k-1)\}-L_{d} u(k-1)\right] \cdot \text { (12) }
$$

\subsection{Estimation of the Depth Related Parameters}

The estimation of the depth $\left(Z_{s}^{()}(k)\right)$ related parameters can be done in multiple ways. In this section, we present some of these algorithms. If the inverse of $\left(s_{z} Z_{s}^{(J)}(k) / f\right)$ is defined $s \zeta_{s}^{(J)}(k)$, then, equations (1) and (2) canbe rewritten as:

$$
\begin{aligned}
& \mathbf{y}_{\mathbf{F}}^{(f)}(k)=2 y_{\mathrm{F}}^{(l)}(k-1)-\mathbf{y}_{\mathbf{F}}^{(f)}(k-2)+\zeta_{3}^{(f)}(k-d) B_{\mathbf{F}_{1}}^{(f)}(k-d) \\
& \Delta T\left(k-d^{())}+B_{\mathbf{F}}^{(j)}(k-d) \Delta R(k-d)+n_{\mathbf{F}}^{(j)}(k)\right.
\end{aligned}
$$

where the vector $\mathbf{n}_{\mathbf{F}}(\boldsymbol{l})(k)$ is a gaussian noise vector with 200 mean and covariance $N^{(J)}(k)\left(n_{F}^{(l)}(k)-N\left(0, N^{(l)}(k)\right)\right)$, and $B_{F_{1}}^{(J)}(k), B_{F}()(k)$ are given by:

$B_{F_{1}}^{(j)}(k)=T\left[\begin{array}{ccc}-1 & 0 & \frac{x^{(f)}(k) s_{x}}{f} \\ 0 & \frac{-s_{z}}{s_{y}} & \frac{y^{(j)}(k) s_{x}}{f}\end{array}\right]$.

$B_{p_{r}}^{(j)}(k)=T\left[\begin{array}{lll}\frac{x^{(j)}(k) y^{(j)}(k) s_{y}}{f} & \frac{-f^{2}-\left(x^{(f)}(k) s_{x}\right)^{2}}{f s_{x}} & \frac{y^{(j)}(k) s_{y}}{s_{x}} \\ \frac{\left.f^{2}+y^{(j)}(k) s_{y}\right)^{2}}{f s_{y}} & \frac{-x^{(j)}(k) y^{(s)}(k) s_{x}}{f} & \frac{-x^{(j)}(k) s_{x}}{s_{y}}\end{array}\right]$,
$\mathrm{AT}(\mathrm{k})=\mathbf{T}(k)-\mathrm{T}(k-1), \quad \boldsymbol{A} \boldsymbol{R}(\boldsymbol{k})=\mathrm{R}(\mathrm{k})-\mathrm{R}(\boldsymbol{k}-1)$.

By defiring $\Delta u_{i}^{(l)}(k)$ and $\Delta u_{f}^{(f)}(k)$ as $\Delta u_{t}^{(f)}(k)=B_{F}^{(f)}(k) \Delta T(k)$ and $\Delta \mathbf{u}_{\mathbf{r}}^{(j)}(k)=\mathbf{B}_{\mathbf{r}}^{(j)}(k) \Delta \mathbf{R}(k)$, equation (13) is transformed into:

$$
\begin{gathered}
\mathbf{y}_{\mathbf{F}}^{(l)}(k)=2 \mathbf{y}_{\mathbf{F}}^{(j)}(k-1)-\mathbf{y}_{\mathbf{F}}^{(l)}(k-2)+\zeta_{s}^{(j)}(k-\phi) \Delta \mathbf{u}_{\boldsymbol{s}}^{(l)}(k-d) \\
+\Delta \mathbf{u}_{\mathbf{r}}^{(j)}(k-d)+\mathbf{n}_{\mathbf{F}}^{(j)}(k) .
\end{gathered}
$$

The final transformation of equation (14) is done by using the vector $\Delta \mathbf{y}_{\mathbf{F}}()(k)$ which is defined as:

$$
\Delta y_{F}()(k)=y_{F}^{(J)}(k)-2 y_{F}^{(J)}(k-1)+y_{F}^{(J)}(k-2)-\Delta u_{r}^{(J)}(k-d) \text {. }
$$

The new form of the equation (14) is:

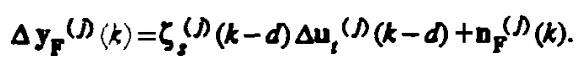

The veotors $\Delta \mathbf{y}_{\mathbf{p}}{ }^{(j)}(k)$ and $\Delta \mathbf{u}_{t}(\boldsymbol{l})(k-d)$ are known at every time instant, while the scalar $\zeta,()(k)$ is continuously estimated. It is assumed that an initial timate $\hat{\zeta}_{s}^{(D)}(0)$ of $\zeta_{s}^{(D)}(0)$ is given and $p^{(D)}(0)$ $=E\left[\left[\zeta_{,}(\lambda)(0)-\hat{\zeta}_{g}^{(j)}(0)\right]^{2}\right\}$ is a positive scalar $p_{0}$. The term $p^{(\lambda)}(0)$ can interpreted as a measure of the confidence that we have in the initial estimate $\hat{\zeta}_{s}^{(\delta)}(0)$. Accurate knowledge of the scalar $\zeta_{3}(\mathcal{H})(k)$ corresponds to a small covariance scalar $p_{\sigma}$. In our examples, $\mathbf{N}(\delta)$ is a constant predefined matrix. To simplify the notation $\mathbf{h}(k)$ is used instead of $\Delta u_{r}(\mathcal{l})(k)$.

The estimation equations are [13]:

$$
\begin{aligned}
& -\xi_{s}\left(\mathcal{s}(k)=+\hat{\zeta}_{s}()(k-1)\right. \\
& { }_{p}(f)(k)=+_{p}()(k-1)+s()(k-1) \\
& { }^{+}{ }_{p}(f)(k)=\left[\left\{{ }^{-p}()(k)\right\}^{-1}+h^{T}(k-d)\{N(f)(k)\}^{-1} h(k-d)\right]^{-1} \\
& \mathbf{k}^{T}(k)={ }^{+} p(d)(k) \mathbf{h}^{T}(k-d)\left\{\mathbf{N}^{(\lambda)}(k)\right\}^{-1} \\
& +\hat{\zeta}_{s}^{(J)}(k)=-\hat{\zeta}_{s}^{(J)}(k)+\mathrm{k}^{T}(k)\left[\Delta \mathbf{y}_{\mathbf{F}}^{(D)}(k)--\hat{\zeta}_{s}^{(J)}(k) \mathrm{h}(k-\delta)\right]
\end{aligned}
$$

where $s^{(f)}(k)$ is a coyariance scalar which corresponds to the white noise that characterizes the transition the states, the superscript $(-)$ denotes the predicted value of a variable, and the superscript $(+)$ denotes its updated value The depth related parameter $\zeta_{j}^{(D)}(k)$ is a time.yarying variable since the target moves in $3 D$ and the camera translates along its optical axis and rotates along the $X$ and $Y$ axis. The estimation scheme of equations (16)-(20) can compensate for the timevarying nature of $<,()(k)$ because it is designed under the assumption that the estimated variable undergoes a random change. One problem is to keep the covariance scalar $p^{(\lambda)}(\boldsymbol{k})$ firite. Solutions for this can be found in [11], In addition. we have implemented other estimation techniques which deal with dime-yasying parameters [9]. In addition to the previous techniques, we propose the use of a more acourate form for the stafe update of $\zeta_{3}^{(l)}(k)$. This form is based on the equation (compurtional delays are included):

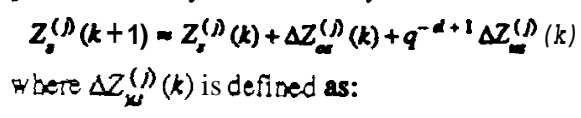

$$
\Delta Z_{m}^{(n)}(k)=-\left\{T_{z}(k)+\left[R_{x}(k) y^{(n)}(k) s_{y}-R_{y}(k) x^{(n)}(k) s_{x}\right\} \frac{Z_{x}^{(j)}(k)}{f}\right] T
$$

and $\Delta Z_{o s}^{(\mathcal{S}}(k)$ is the change in depth induced by the motion of the tanget. It is assumed that $\boldsymbol{\Delta} \boldsymbol{Z}_{o s}^{(\mathcal{S})}(\boldsymbol{k})$ does not change significantly between two time instances. The term $\Delta Z_{u s}^{(f)}(k)$ is created by the motion of the camera and is derived by an a gebrajc computation described in [9]. Equation (21) provides an approximation of the change in the feartre's depth $Z_{s}^{(N)}(k)$ twen two time irstances given the fearure's image coordinates and the camera motion. This equation can be rewritten as:

$$
Z_{s}^{(n)}(k)=2 Z_{a}^{(n)}(k-1)-Z_{a}^{(n)}(k-2)+\Delta Z_{m}^{())}(k-d)-\Delta Z_{m}^{())}(k-d-1) \text {. }
$$


By inverting the terms of the previous equation (22), the following equation is derived:

$$
\begin{aligned}
& \zeta_{f}^{(\lambda)}(k)=\zeta_{t}^{(j)}(k-1) /\left(2-\frac{\zeta_{t}^{(\prime)}(k-1)}{\zeta_{z}^{(j)}(k-2)}\right. \\
& \left.+\zeta_{j}^{(n)}(k-1) \frac{s_{x}}{f}\left[\Delta Z_{w \infty}^{(n)}(k-d)-\Delta Z_{m}^{(n)}(k-d-1)\right]\right\}
\end{aligned}
$$

where

$$
\Delta Z_{z}^{(j)}(k)=-\left\{T_{z}(k)+\left[R_{x}(k) y^{(j)}(k) s_{y}-R_{y}(k) x^{(j)}(k) s_{x}\right] \frac{s_{x}}{\zeta_{z}^{(j)}(k)}\right\} T .
$$

If we substitute the values of $\zeta_{s}^{())}(k)$ with their estimates, (23) will be transformed into:

$$
\begin{aligned}
& -\zeta_{f}^{(j)}(k)=+\zeta_{j}^{(j)}(k-1) /\left(2-\frac{+\zeta_{z}^{(j)}(k-1)}{+\zeta_{z}^{(j)}(k-2)}\right.
\end{aligned}
$$

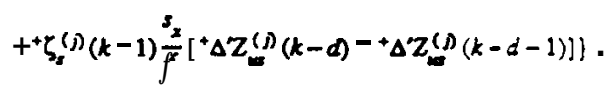

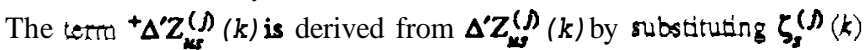
with $+\zeta_{z}^{(\Omega)}(k)$. In addition, equation (17) should be modified to inoorporate the new equation for the updates of states. These estimation schemes require the estimation of one parameter per festure-point and therefore, the real-time implementation of the estimation scherre is feasible. In addition, we have implemented an estimation scheme that computes two parameters per feature point. This scheme is a variation of the previous estimation scheme and separately esirestes the depth relared parameiers $\left(f /\left(s_{x} Z_{s}^{(j)}(k)\right)\right)$ and $\left(f /\left(s_{y} Z_{s}^{(j)}(k)\right)\right)$ in the $X$ and $Y$ directions on the image plane. In theory, this fomulation can estimate the depth related parameters more accurately.

The matrices $\mathbf{B}_{\mathbf{F}_{\mathbf{I}}}{ }^{(j)}(k)$ and $\mathbf{B}_{\mathbf{F}}{ }_{\mathbf{z}}(k)$ are transformed and decomposed as follows:

$$
\begin{aligned}
& \mathbf{B}_{\mathbf{F}_{a}}{ }^{()}(k)=T \quad\left[-1 \quad 0 \quad \frac{x^{(j)}(k) s_{x}}{f} 1\right. \\
& B_{F_{0}}(k)=T \text { I } 0 \quad \frac{-s_{x}}{s_{y}} \quad \frac{y^{(s)}(k) s_{y}}{f} \text { ) } \\
& \mathrm{B}_{\mathrm{F}_{n}}(j)(k)=T 1 \quad \frac{x^{(j)}(k) y^{(j)}(k) s_{y}}{f} \quad \frac{-f^{2}-\left(x^{(f)}(k) s_{x}\right)^{2}}{f s_{x}} \quad \frac{y^{(f)}(k) s_{y}}{s_{x}} 1 \\
& \mathrm{~B}_{\mathrm{F}_{n}}{ }^{(n)}(k)=T\left[\frac{\left.f^{2}+\sigma^{(j)}(k) s_{y}\right)^{2}}{f s_{y}} \quad \frac{-x^{(f)}(k) y^{(f)}(k) s_{x}}{f} \quad \frac{-x^{(f)}(k) s_{x}}{s_{y}}\right] \text {. }
\end{aligned}
$$

The subscript $\mathrm{i}$ denotes the $\mathrm{X}$ or $\mathrm{Y}$ direction. The estimation equations for each fearure point art $(i=1,2)$;

$$
\begin{aligned}
& -\hat{\zeta}_{s i}^{(j)}(k)=+\hat{\zeta}_{s i}^{(D)}(k-1) \\
& -p_{i}^{()}(k)=+p_{i}^{(n)}(k-1)+s_{i}^{(n)}(k-1) \\
& { }^{+} p_{i}^{(D)}(k)=\left[\left\{{ }^{-} p_{i}^{(D)}(k)\right\}^{-1}+h_{i}(k-d)\left[n_{i}^{(D)}(k)\right]^{-1} h_{i}(k-d)\right]^{-1} \\
& x_{i}(k)={ }^{+} p_{i}^{(f)}(k) h_{i}(k-d)\left\{n_{i}^{(f)}(k)\right\}^{-1} \\
& +\hat{\zeta}_{s i}^{(D)}(k)=-\hat{\zeta}_{s i}^{(l)}(k)+x_{g}(k)\left[A y_{F i}(l)(k)--\hat{\zeta}_{s i}^{(J)}(k) h_{i}(k-a)\right]
\end{aligned}
$$

where $\Delta y_{g i}(\lambda)(k)$ and $h_{i}(k)$ denote the $X$ or $Y$ components of the vectors $A \mathbf{y}_{\mathbf{F}}{ }^{(\lambda)}(k)$ and $\mathrm{h}(\mathrm{k})$, respectively, and $\hat{\zeta}_{s i}^{(D)}(k)$ is the estimated value of either the term $\left(f /\left(s_{x} Z_{z}^{()}(k)\right)\right)$ or the term $\left(f /\left(s_{y} Z_{z}^{(l)}(k)\right)\right)$. In practice, the experimental results from the implementation of this estimation scheme prove to be comparable with the results of the first estimation scheme. Some researchers [3] propose the use of an adaptive scheme that extimates all the elements of the block matrix B (k) an-line. This approach is computationally expensive and not necessary.

\subsection{Implementation Issues and Robot Controllers}

In the experiments, we are forced to bound the input signals in order to avoid saturation of the actuators. After the computation of the translational $\mathbf{T}(k)=\left(T_{x}(k), T_{y}(\&), T_{z}(k)\right)^{T}$ and rotational velocity vectors $R(k)=\left(R,(k), R_{y}(k), R_{z}(k)\right)^{T}$, we Limit the input Signals by performing seyeral steps that arc described in [9]. Thus. the vectors $\mathbf{T}(k)$ and $\mathbf{R}(k)$ are transformed to $T^{\prime}(k)$ and $R^{\prime}(k)$, respectively,

After computing the translational velocity vector $\mathbf{T}^{\mathbf{N}}(\boldsymbol{k})$ and the rotational velocity vector $R^{\prime}(k)$ with respect to the camera frame $R_{s^{\prime}}$ we transform it to the end-effector frame $R$, with the use of the transformation $\boldsymbol{T}_{\text {, }}$. The transformed signals $\boldsymbol{m}$ fed to the robot controller of the PUMA which acts as the traces. We use the Unimation controllers which are inferiaced to our system through multiple lronics IV.3230 CPU boards. The Alter lime is used and the desired trajectory in cartesian space is updared $28 \mathrm{~ms}$. W e arc currently in the prosess of substituting the Unimation controllers with Trident boards which can be programmed in $\boldsymbol{C}$. Finally, the whole system runs under the CHIMERA II real-tige oferaing system [14], The hardware configuration of the TROIKABOT system is described in [9].

The next section describes the experimental results of our algorithms on the TROIKABOT multi-robotic system.

\section{Experimental Results}

The algorithms have been verified by nertoming a number of experiments on the TROIKABOT robotic system [15]. A camera is mounted on the end-effector of one of the PUMAs which acts as the tracker. The other PUMA holds a target and moves it accordingly. The real images are $492 \times 510$ and art quantized to 256 gtay levels. The camera's pixel dimensions ar: $s_{x}=0.011 \mathrm{~mm}$ pixel and $s_{y}=0.013 \mathrm{~mm} / \mathrm{pixel}$. The focal length of the camera is $16 \mathrm{~mm}$ and the objects move with full 3-D motion. To initial depth of the objects' center of mass with respect to the canter frame $Z$, is $290 \mathrm{~mm}$. The maximum permissible translational velocity of the end-effector of the hacking robot is $10 \mathrm{~cm} / \mathrm{sec}$ and each of the components of the endefiector's rotational velocity (roll. pitch, yaw) is not allowed to exed $0.3 \mathrm{rad} / \mathrm{sec}$. The objective is to move the manipulator so that the image projections of certain features of the moving object move to some desired image positions or stay at their initial positions. The objects used in the servoing examples cre books, peocils, or any item with distinct fearures. The user uses the mouse to select fearures of the object to be used in tracking. Then, the system evaluates on-line the quality of the features, based on the confidence measures described in [6]. The same operation can be done automatically by a computer process that runs once and noeds 2 or 3 minutes, depenting on the size of the interest operators which are used. The four best fearures are selected and used for the robotic visual servoing task The size of windows is $8 \times 8$ while the search area is 64x64. The maximum displament sampling period $T$ that can be detected is $\mathbf{2 8}$ pixels. The SSD algarith has beer implemented by using the pyramidal structure described in [9] An interesting solution to the antomatic detection and selection of point fearures has been proposed by Tomasi and Kanade [16]. We arc currently investigating the polential of this approach as an altematye to our algorithms for the selection of the best feature points.

Experimental resulis are presented in Figures 1 through 6 . The gains for the controllers are $\mathbf{Q}=0.9 \mathbf{I}_{\mathbf{B}}, \mathbf{L}=\mathbf{0}$, and $\mathbf{L}_{\mathbf{d}}=\operatorname{diag}\{0.04,0.04,1.0$, $\left.5 \times 10^{5}, 5 \times 10^{5}, 5 \times 10^{5}\right\}$. The diagonal elements of the $\mathrm{Q}, \mathrm{L}$, and $\mathrm{L}_{\text {r }}$ can vary by a factor of between 2 and 3 and the system will continue to track successfully. The delay factor $d$ is 2 . The vector $\mathbf{y}^{*}(k)$ is given every instant of time $\boldsymbol{k}$ by the dation $\mathbf{y}^{*}(\boldsymbol{k})=\boldsymbol{y}(0)$. This implies that the objective of our scheme is to the fearures ar their initial positions during the motion of the target. 
The computation of the $\left\langle\hat{\mathbf{B}}^{T}(\mathbf{k}) \mathbf{Q} \hat{\mathbf{B}}(k)+L+L_{d} \Gamma^{-1}\right.$ ratrix is done on a Heurikon 68030 board. The technique used is the same as the one described in [10]. The total computation time (image processing and control calculations) of $T^{\prime}(k)$ and $R^{\prime}(k)$ is approximately $220 \mathrm{~ms}$. The knowledge of the depth $Z$, is assumed to be inaccurate. For all the fearures, $\zeta_{s}^{(n)}(0)$ is initialized to 3.63 and $\rho^{(\nu)}(0)$ is 0.1 .

In the example depicted in Figures 1 through 6 . the performance of the control and estimation algorithms is illustrated. The target's trajectory is plotted with restest to the frame $R$, which is attached to the target at the time instant $k=0$. At the same instant, the $Z$ axis of the $R_{t}$ frame is aligned with the optical axis of the camera The extimation scheme which is used estimates one parameter feanure point, thus, four parameiers estimated in total. The forgetting factor is 0.99 . The measured deviations of the features from their desired positions appear noisy. The fact that the errors on the image plane are bounded guarantees that the erors arc within the search range of the SSD algorithm, thus. the SSD algorithm can accurately measure the features' positions. The errors reach a maximum value when the target changes its trajectory sharply. The control and estimation algorithms compensate quickly and after $\mathbf{1 0}$ seconds the errors arc reduced. The error in the $Z$ direction is large. The reason is that the noisy measurements, the camera geometry, and the experimental serup make the accurate computation of the tracking motion in the $\mathbf{Z}$ direction (along the qtical axis of the camera) difficult. Another interesting observation is that there is a small error in pitch even though the is no pitch component in the target's motion. This phenomeron ocaurs since then is a strong coupling betreen the pitch component and the $\mathbf{X}$ translational component of the tracking motion The same is twe for the yaw component and the $Y$ translational component of the tracking motion. In other words, the tracking system tries to tradk $\mathbf{X}$ translational or $\mathbf{Y}$ translational motion of the target with the rostional degrees of freedom, $R_{y}$ or $R_{z}$ respectively. Numerically, this implies that the conditio? number $c$ $\left(c=\sigma_{\max } / \sigma_{\min }\right.$, a ratio of singular values) of the matrix $B(k)$ is large. Appropriate selection of the feature points and the relative position of the camera with respect to the target can minimize the condition number. If the relative distance of the camera (assuming the same focal length for the camera) from the target is more than 2 meters, the condition number omes too large and tracking is impossible. In addition, full tracking is impossible when the farr feature points arc close to each other, or if they arc very close to the piercing point.

\section{Conclusions}

In this papa, we examined the problem of robotio visual tracking of full 3-D motion (three translations and three rotations) by a monocular robotic tracker. A camera is mounted on the end-effector of the robotic device and provides visual information about the motion of the target. The detection of motion is based on an optical flowtechnique called Sum-of-Squared Differences (SSD) optical flow. This algorithm, which has been implemented in a pyramidal scheme for computational efficiency, provides the displacement vector of certsin selected fearures of the target. Under the general guidelines of the combrolled active vision framework which was introduced in [7], we combine these measurements with appropriate control and estimation techniques. Adaptive control techniques arc introduced to compensate for uncer. tainties in the model, unknown depth related parameters, and computational delays. The computational burden is reduced by estimating only one $\boldsymbol{\alpha}$ two parameters per feature point. Our algorithms do not require accuratecslibration of the workspace, and thus, can be efficiently used in assembly lines in order to track moving items. In addition, these algorithms make possible autonomous satellite docking and recovery. The algorithms were extensively tested in several experimests which wen the TROIKABOT muld-robotic system. The reat time experiments show the feasitility and efficiency of our algorithms. In gentral, these algorithms show thet morocular vision in conjunction with efficieat motion of the vision sensor and adaptive control algorithms can be a viable alterrady to standard stereo vision techniques.

Some of the areas for funde researah which we arc currently considering include the use of more elaborate MDMO adaptive control techDiques than those that have been implemented, the computational improvement of our algorithms. and the incroduction of algarithms for asing dges as the of motion information. We se currently pussung the use of "makes"for contour serving, the application of idsolye algorithms to model-based visual tracking and servoing, and the derivation of depth maps strough appropriate motion of the robotserrem in conjunction with simple adaptive filtering techniques.

\section{Acknowledgements}

This research was supported by the Defense Advanced Research Projects Agency through ARPA Order Number DAAA-21-89C-0001. and by the U.S. Army Research Office through grant Number DAAL 03-91-G-0272. The views and conclusions contained in this document the the of the authors and should not be interpreted as representing the official policies, either expressed or implied, of the funding agencies.

\section{References}

1. P.K. Allen, "Real-time maim tracking using spatio-temporal filters" Pra.DARPA Image Understanding Workshop, 1989, pp. 695-701.

2. F. Chumette, P. Rives, and B. Espian, "Positioning of a raboe with respect to a object, tracking it and extimating its velocity by visual servoing", Proc. of the IEEE Int. Conf. on Robotics and Automation, April 1991, pp. 2248-2253.

3. J.T. Feddema and C.S.G. Lee, "Adaptive image feanure prediction and control f a viaul tracking with a berd-eye coordinated camar'", IEEE Trans. on Systems, Man and Cybernetics, Vol. 20, No. 5,1990, pp. $1172-1183$.

4. A.J. Ka vo and N. Houshngi, "Real-time vision feedbeck for servoing of a robotic manipulator with" self-muing controllex", IEEE Trans. on Systems, Man and Cybernetics, Vol 21, No. 1, 1991, pp. 134-142.

5. ED. Dicknanns, B. Mysliwetz, a d T. Christims, "An integrated epatio-lemporal epproech to automatic visual guidance of autonomous vehickes", IEEE Trans. on Systems, Man, and Cybernetics, Vol. 20, No. 6, 1990,pp. 1273-1284.

6. N. Pepenikolopoulos, P.K. Khosla, a d T. Kasede, "Vision and control tochniques f a robotic visual tracking", Proc. of the IEEE IN. Conf. on Robatics and Aubmation, 1991,pp. 857-864.

7. N. Papenikolopoulos, P.K. Khosh, and T. Kanade. "Adaptive robotic visul unacing", Proc. of the 1991 American Control Conference, June 1991, pp. 962--07.

8. N.P. Pannikolopoulos and PX. Khosle "Fentume besed robocic visual trecking of 3-D transitional mocion". Proc. of the 30th IEEE CDC, Brighton, UK, Docember 1991, pp. 1877-1882.

9. N.P. Papmirolopoulos, Controlled active vision, $\mathrm{PhD}$ dissertation, Department of Electrical and Computer Engineering, Camegie Mellon University, August 1992

10. N.P. Papanikolopoulos and P.K. Khosla, "Robotic visual servoing around a static terget: an exmple of controlled ective vision", Roc. of ihe 1992 American Control Conference, June 1992,pp. 1489-1494.

11. G.C. Goodwin and K.S. Sin, Adaptive filtering, prediction and control, Prentice-Hall, inc., Englewood Cliffs, New Jersey 07632, Information and Systems Science Series, Vol. 1,1984.

12 F.L. Lewis, Optimal control, John Wiley \& Sons, New Yak. 1986.

13. P.S. Maybeck, Slochastic madels, estimation, and control, Academic Press, London, 1979. 
B. Stewart, DE Schmin, and P.K. Khosih, "Implementing real-time robotic systems using CHIMERA II", Proc. of 1990 IEEE Int. Conf. on Robotics and Automation, Cincinnati, Ohio, May 1990, pp. 598-603.

15. FE. Acker, I Ince, and B.D. Otinger, "TROIKABOT - A multi-ermed essembly robox". Proc. of the Robots 9 Conference, Detroi, MI, June 3-6 1985.

16. C. Tomesi and T. Kenede, "Detection and trecking of point fentures", Tech. report CMU-CS-91-132, Carnegie Melloo University, School of Computer Science, 1991.

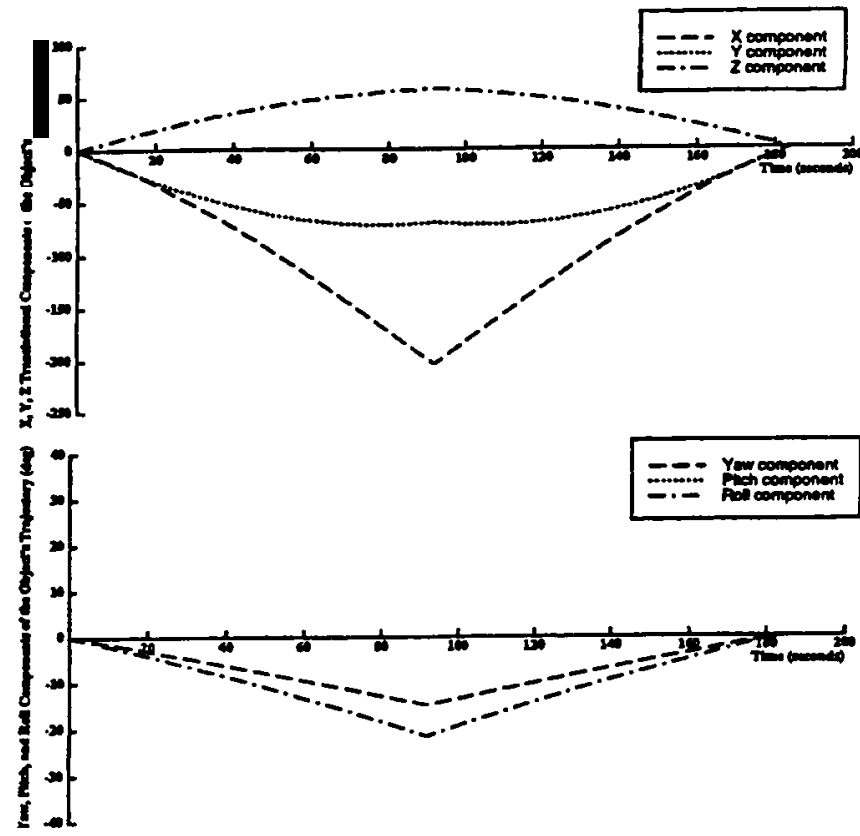

Flgure 1: Translational and rotational trajectories of the moving object with respect to its initial pose (experimental).
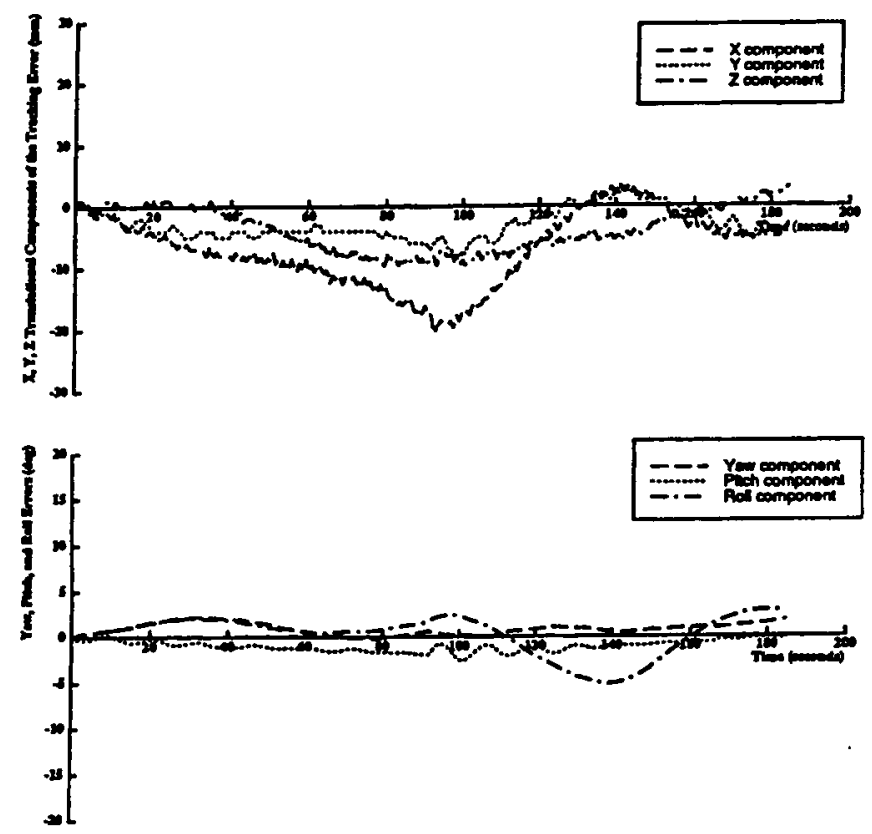

Flgure 2: Translational and rocational tracking errors in the previous example (experimental)

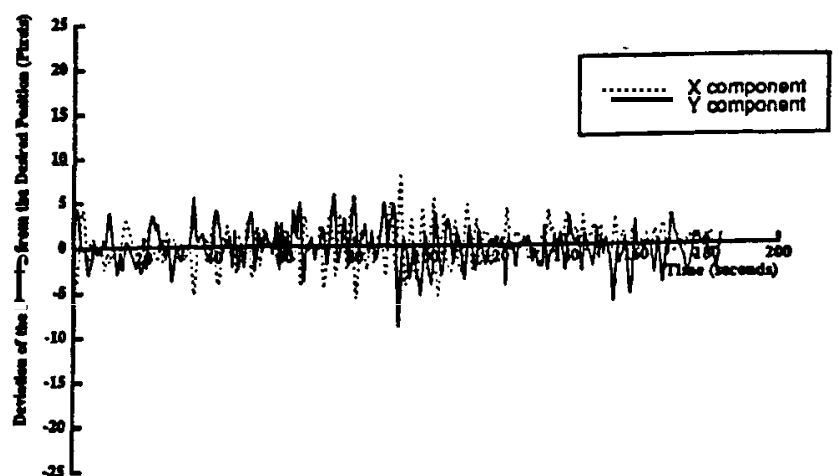

Figure 3: Devimion of fenture A from its desired position in the previous exmmple (experimental).

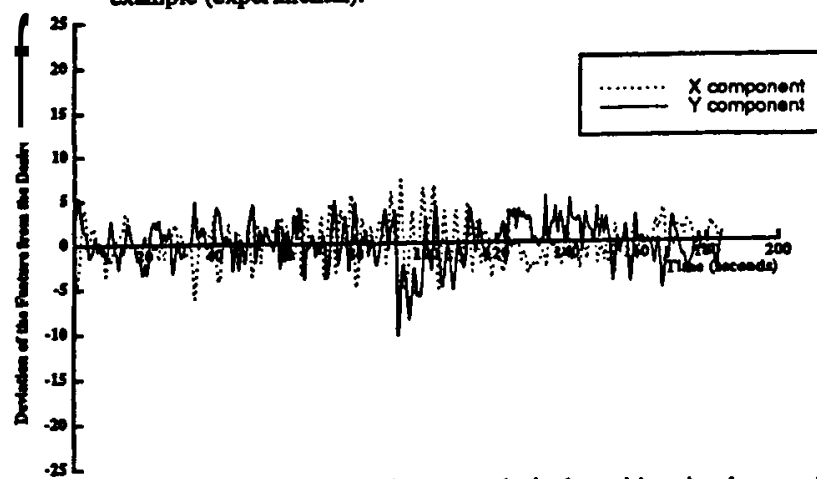

Migure 4: Devicion of fenture B from its desired position in the previou exmple (experimenta).

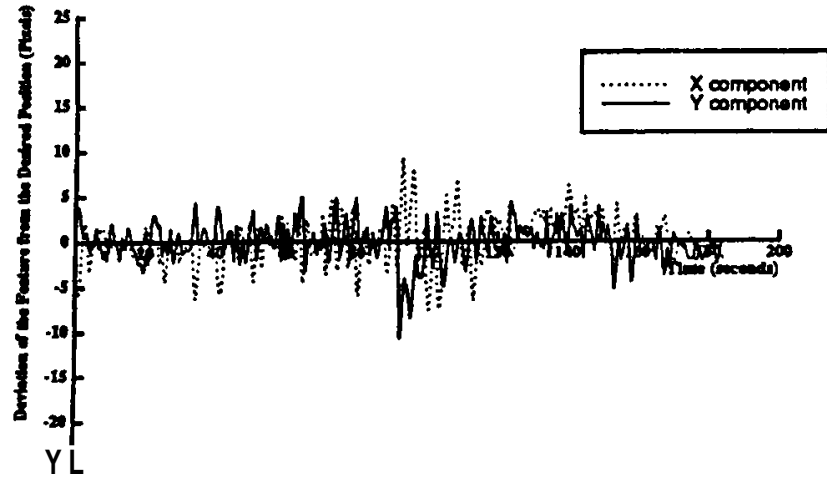

Fpure S: Devintion of feanure $C$ from its desired position in the previous exemple (experimentunt.

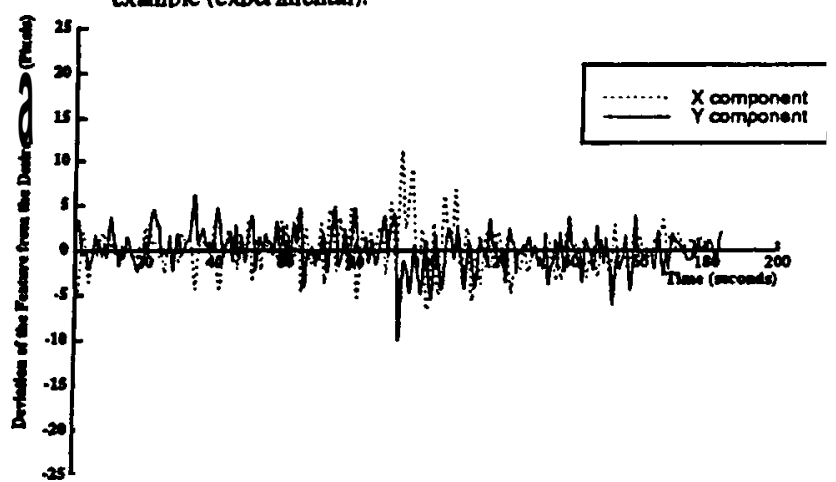

Flgure 6: Deviation of feature D from its desired position in the previous example (experimenta). 